\title{
Effect of Ultrasound on Transdermal Drug Delivery to Rats and Guinea Pigs
}

\author{
D. Levy, ${ }^{\star}$ J. Kost, ${ }^{\ddagger}$ Y. Meshulam, ${ }^{*}$ and R. Langer ${ }^{\$}$ \\ *Israel Institute for Biological Research, Ness-Ziona, Israel; ${ }^{\ddagger}$ Department of Chemical Engineering, Ben-Gurion University, \\ Beer-Sheeva, Israel; ${ }^{\S}$ Department of Chemical Engineering, Massachusetts Institute of Technology, Cambridge, Massachusetts 02139
}

\begin{abstract}
The effect of therapeutic range ultrasound (1 MHz) on skin permeation of D-mannitol, a highly polar sugar alcohol, inulin, a high molecular weight polysaccharide and physostigmine, a lipophilic anticholinesterase drug was studied in rats and guinea pigs. D-Mannitol and inulin are totally and rapidly excreted, once they have penetrated through the skin into the blood stream, permitting direct in vivo monitoring. For evaluating skin penetration of physostigmine the decrease of whole blood cholinesterase was measured. Ultrasound nearly completely eliminated the lag time usually associated with transdermal delivery of drugs. 3-5 min of ultrasound irradiation (1.5 $\mathrm{W} / \mathrm{cm}^{2}$ continuous wave or $3 \mathrm{~W} / \mathrm{cm}^{2}$ pulsed wave) increased the transdermal permeation of inulin and mannitol in rats by 5-20-fold within 1-2 h following ultrasound application. Ultrasound treatment also significantly increased $(P<0.05)$ the inhibition of cholinesterase during the first hour after application in both physostigmine treated rats and guinea pigs: while in control guinea pigs no significant inhibition of cholinesterase could be detected during the first $2 \mathrm{~h}$ after application of physostigmine, the ultrasound treated group showed a $15 \pm 5 \%$ (mean \pm SEM) decrease in blood cholinesterase $1 \mathrm{~h}$ after ultrasound application. For physostigmine-treated rats the level of cholinesterase inhibition $1 \mathrm{~h}$ after ultrasound application was $53 \pm 5 \%$ in the ultrasound-treated group and $35 \pm 5 \%$ in the controls.
\end{abstract}

\section{Introduction}

Topical administration of drugs has been used for many years, but only recently has controlled administration of systemic drugs through the skin (transdermal drug delivery) become a clinically accepted procedure. While the earliest indications that transdermal delivery might be useful date back to the 1960s (1), the first transdermal therapeutic system (for scopolamine), was introduced in 1981. Recently, several other transdermal systems (for nitroglycerine, estradiol, clonidine) have been developed (2). However, while transdermal delivery of drugs is an alluring concept, it has drawbacks that limit its scope. Relatively few drugs penetrate the skin at rates high enough to yield therapeutic levels in the plasma, and the lag time for establishing a stable concentration of the drug in the

Address reprint requests to Dr. Langer, Massachusetts Institute of Technology, E25-342, 77 Massachusetts Avenue, Cambridge, MA 02139.

Received for publication 17 August 1987 and in revised form 7 February 1989.

J. Clin. Invest.

(C) The American Society for Clinical Investigation, Inc.

$0021-9738 / 89 / 06 / 2074 / 05 \$ 2.00$

Volume 83, June 1989, 2074-2078 circulation may be several hours or even days (3). For example: transdermal systems for clonidine proposed for antihypertensive therapy have been reported to reach therapeutic steady state levels only after one to two days (3); scopolamine which is used for treating motion sickness is also effective only several hours after application (4). In order to enhance penetration of drugs through the skin it is possible to use chemical enhancers or to manipulate skin permeability by external means such as iontophoresis (5). External modulation of skin permeability may allow better control of drug release rates than the use of chemical enhancers, as it can be stopped and reapplied according to need. The actual rate of administration of a systemic drug from a transdermal therapeutic system is related to the rate of drug release from the therapeutic system and the rate of drug permeation through the skin (6). The lag time phase may also be attributed to both the skin and the transdermal system, as it takes some time for the drug to migrate from the therapeutic system and across the skin. We have previously shown that ultrasound can enhance the release rates of drugs through solid polymers (7), and in the current study, examined the effect of ultrasound on skin permeation of drugs with special attention to the lag time period. Ultrasound has previously been explored for treating localized skin conditions $(8)$, and for delivering drugs to inflamed joints $(9,10)$. However, there is limited quantitative information on the effect of ultrasound on the delivery and the lag time phase of transdermally administered drugs.

In the current study we report that ultrasound nearly completely eliminates the lag time associated with transdermal delivery, and in some cases increases the overall drug penetration through the skin. Three different substances and two animal models, rats and guinea pigs, were used. The molecules evaluated were D-mannitol, a highly polar sugar alcohol, inulin, a large molecular weight polysaccharide, and physostigmine, a lipophilic molecule. D-Mannitol and inulin are totally and rapidly excreted $(11,12)$ permitting direct in vivo monitoring of the labeled drug once it penetrated through the skin into the blood circulation. Physostigmine is a reversible inhibitor of cholinesterase, used for its anticholinesterase action in glaucoma (13), in tricyclic antidepressant poisoning (13) and in the treatment of Alzheimer's disease (14). The biological effect of physostigmine was determined by measuring the decrease of whole blood cholinesterase of physostigmine-treated animals. The ultrasonic unit applied was a therapeutic ultrasound system approved for human use.

\section{Methods}

Materials. The test solutions were: $\mathrm{D}-\left[{ }^{3} \mathrm{H}\right]$ mannitol (New England Nuclear, Boston, MA), $1 \mathrm{mCi} / \mathrm{ml}$ in ethanol water solution 9:1, saturated with unlabeled D-mannitol (Sigma Chemical Co., St. Louis, MO); $\left[{ }^{3} \mathbf{H}\right]$ inulin (New England Nuclear) mixed with unlabeled inulin and dissolved in warm water to make a $1-\mathrm{mCi} / 5 \mathrm{ml}$ saturated solution; and physostigmine base (Sigma) in ethanol ( $15 \%$ or $30 \% \mathrm{wt} / \mathrm{vol})$. We 
did not use saturated solutions of physostigmine in order to avoid too high a cholinesterase inhibition in control animals.

In vitro studies. A transport cell composed of two compartments $(48 \mathrm{ml})$ each separated by the membrane of interest was used to obtain the permeability data. The membranes evaluated were cellulose $(\mathrm{Cu}-$ prophan) and microporous polypropylene films (Celgard; Celanese Corp., New York). The permeating molecules detected were: $\mathrm{NaCl}$, $\mathrm{KIO}_{3},\left(\mathrm{NH}_{4}\right)_{2} \mathrm{~S}_{2} \mathrm{O}_{8}$, urea (Merck \& Co., Inc., Rahway, NJ) and bovine serum albumin (BSA) (Sigma). In these experiments an ultrasonic probe (Vibra Cell 250; Sonics and Materials, Danbury, CT) was immersed into the upstream compartment and the solution was exposed to ultrasound. The downstream compartment solution was circulated (Ismatec pump 7620; Zurich, Switzerland) through a conductivity cell (El-Hamma EHT-24, TH-2400; EL-Hamma, Israel) to detect salt conductivity, or a flow-through spectrophotometer (Spectronic 1001; Bausch \& Lomb, Inc., Rochester, NY) at $220 \mathrm{~nm}$ for BSA detection. Urea was detected by taking aliquots from the downstream compartment based on the assay developed by Marsh et al. (15). In several permeability experiments instead of an ultrasonic probe, a stirrer (RGL-100; Heidolph, Kelheim, West Germany) was immersed in the upstream compartment and permeability was evaluated at defined mixing rates. To evaluate the effect of temperature on permeability, experiments were performed in a thermostated environment set to defined temperatures (model 3550 ịncubator; Lab-Line Instruments, McGaw Park, IL). In several other permeability experiments the solution in the transport cell was degassed just before the permeability experiment (vacuum of $60 \mathrm{~mm} \mathrm{Hg}$ for $15 \mathrm{~min}$ ).

In vivo drug and ultrasound application. At $30 \mathrm{~min}$ before drug application the hair of dorsal test sites of the experimental animals (Sprague-Dawley rats, 200-300 g each, or albino guinea pigs of a local strain, Dunkin-Hardly origin, 300-400 g each) was closely clipped using an electric clipper, and the skin was washed with a mild detergent (Proseptyl Bactericide; Sicca, Holon, Israel) to effect wetting by the ultrasonic coupling gel (EMS Therasonic coupling medium). Test solutions of $20 \mu \mathrm{l}(20 \mu \mathrm{Ci})$ of $\mathrm{D}-\left[{ }^{3} \mathrm{H}\right]$ mannitol, $50 \mu \mathrm{l}(11 \mu \mathrm{Ci})$ of $\left[{ }^{3} \mathrm{H}\right]$ inulin or 6-12 $\mu$ l of physostigmine were applied to test sites on the upper back of rats or guinea pigs, $(20 \mu \mathrm{l} / \mathrm{kg}$ of $15 \% \mathrm{wt} / \mathrm{vol}$ physostigmine in ethanol for rats, and $30 \mu \mathrm{l} / \mathrm{kg}$ of $30 \% \mathrm{wt} / \mathrm{vol}$ of physostigmine in ethanol for guinea pigs), followed by the ultrasonic gel. Ultrasound energy, 1.5 $\mathrm{W} / \mathrm{cm}^{2}$ continuous wave $(\mathrm{CW}),{ }^{1}$ or $3 \mathrm{~W} / \mathrm{cm}^{2}$ pulsed wave $4: 1$, was generated by an ultrasonic transducer (1 MHz Therasonic Double Five, model 14A; Electro Medical Supplies, Greenham, Wantage, Ltd., Oxfordshire, England) applied to the treated area for $3 \mathrm{~min}$ for a $\mathrm{CW}$ or $5 \mathrm{~min}$ for a pulsed mode (CW ultrasound was applied for a shorter time and lower intensity to avoid excess heating). Skin temperature was recorded immediately after ultrasound application by placing a temperature probe (Tele Thermometer model 46 TUC; Yellow Springs Instruments, Inc., Yellow Springs, $\mathrm{OH}$ ) on the surface of the treated skin. The treated area was covered by Blenderm surgical tape (3M Corp., St. Paul, MN).

Permeability evaluation. The D- $\left[{ }^{3} \mathrm{H}\right]$ mannitol and $\left[{ }^{3} \mathrm{H}\right]$ inulin treated animals were housed in metabolic cages for the duration of the experiment or catheterized to obtain more reliable short term data. Urine was collected at several time points, $10 \mathrm{ml}$ of scintillation fluor (Aquasol 2; New England Nuclear) was added, and the radioactivity was measured by liquid scintillation counting (Tri-Carb 4530; Packard Instruments, Downers Grove, IL).

For the catheterization, the rats were anesthetized with methoxyflurane (Pitman Moore Inc.), and their abdominal and cervical fur were shaved and cleansed. A 2-cm long midline abdominal incision was made through the abdominal musculature. The bladder was exposed, a nick was made in the bladder top, the catheter (PE-50, 7411; Intramedic Clay Adams, Parsippany, NJ) was inserted in the bladder and secured by 4-0 Dexon suture. After urine flow was verified, the abdominal musculature was sutured (4-0 Dexon) as well as the skin

1. Abbreviations used in this paper: $\mathrm{ChE}$, cholinesterase; $\mathrm{CW}$, continuous wave; US, ultrasound. incision (4-0 nylon). Test solutions of $\mathrm{D}-\left[{ }^{3} \mathrm{H}\right]$ mannitol or $\left[{ }^{3} \mathrm{H}\right]$ inulin were applied to the skin, followed by ultrasound application as described above. Urine was collected every 15-30 min for the first $2 \mathrm{~h}$ and at several additional times during the experiment, and the radioactivity was measured.

For evaluating the effect of ultrasound on the transdermal delivery of physostigmine, blood samples of physostigmine treated rats or guinea pigs were obtained at specific times by cardiac puncture and the decrease of whole blood cholinesterase (ChE) activity was assayed by the Johnson method (16).

Controls. Control animals in each experiment were treated by the procedure described above moving the ultrasonic applicator head over the treated area for 3-5 min, with the power level at the applicator set to zero.

To check for a possible decomposing effect of the ultrasound treatment on the applied D-mannitol, urine samples of the ultrasound treated rats and controls were compared by thin-layer chromatography on silica plates (Kieselgel 60; Merck) developed in $t$-butanol/pyridine/ water $(6: 4: 3)$ or in $t$-butanol/formic acid/water (33:50:17) and visualized by phosphomolybdic acid reagent. The developed silica gel plates were cut to several sections according to the locations of the spots, and the pieces were placed in scintillation vials. $1 \mathrm{ml}$ of distilled water was added to each vial to dissolve the mannitol, then $10 \mathrm{ml}$ of Aquasol scintillation liquid was added and the radioactivity measured.

We also checked the ultrasound effect on ChE inhibition of blood previously inhibited by physostigmine in vitro. 1-ml samples of heparinized blood collected from rats were incubated at $37^{\circ} \mathrm{C}$ in the presence of $5 \times 10^{-9}-5 \times 10^{-8} \mathrm{M}$ physostigmine. After steady state was reached $(\sim 30 \mathrm{~min}$ ), samples were placed in a water bath (room temperature) in which an ultrasonic probe was inserted for $5 \mathrm{~min}(3$ $\mathrm{W} / \mathrm{cm}^{2}$ pulsed wave). The level of cholinesterase inhibition before and after the ultrasound treatment was recorded (Table I).

Histology. Rats exposed to ultrasound, as described above (5 min 3 $\mathrm{W} / \mathrm{cm}^{2}$, pulsed wave), were killed using an overdose of carbon dioxide. Sections $(5 \mathrm{~mm})$ were prepared using hematoxylin and eosin staining.

Statistical analysis. Values are expressed as mean \pm SEM. Student's $t$ test was used for statistical analysis.

\section{Results}

In vivo the pronounced effect of ultrasound on transdermal permeability was observed for all three molecules studied. During the first $2 \mathrm{~h}$ the secretion rate of radiolabeled mannitol was 20-fold higher in the ultrasound treated group than in the controls (Fig. 1). In the inulin experiment (Fig. 2), the experimental group had five times more excreted radioactive drug than the control group during the first hour.

In order to eliminate the possibility that the excess radioactivity observed in the excretion of ultrasound-treated rats was

Table I. The Concentration Dependence of Inhibition of Rat Blood Cholinesterase by Physostigmine

\begin{tabular}{ccc}
\hline $\begin{array}{c}\text { Concentration of } \\
\text { physostigmine }\end{array}$ & \% of ChE inhibition & $\begin{array}{c}\text { \% of ChE inhibition } \\
\text { after ultrasound pulsing }\end{array}$ \\
\hline$n M$ & & \\
5 & $17 \pm 3$ & $18 \pm 3$ \\
10 & $29 \pm 4$ & $24 \pm 4$ \\
20 & $45 \pm 2$ & $40 \pm 7$ \\
30 & $58 \pm 2$ & $53 \pm 3$
\end{tabular}

The blood samples were incubated with physostigmine for $30 \mathrm{~min}$ at $37^{\circ} \mathrm{C}$, then placed in a water bath (room temperature) and pulsed with US probe for $5 \mathrm{~min}\left(3 \mathrm{~W} / \mathrm{cm}^{2}\right.$ pulsed wave). Each value is the mean \pm SEM of three experiments. 


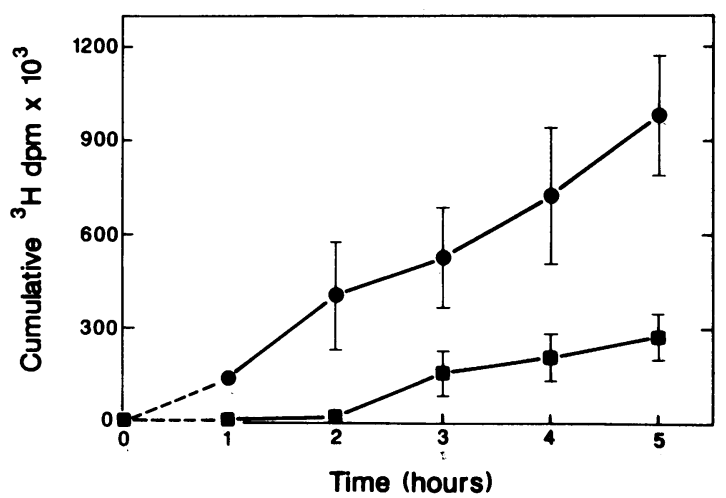

Figure 1. Rats' cumulative radioactive secretion after topical application of $20 \mu \mathrm{l}$ of saturated solution of D-mannitol containing $20 \mu \mathrm{Ci}$ D$\left[{ }^{3} \mathrm{H}\right]$ mannitol. $\bullet$, Ultrasound (US) treated rats. $n=4 .\left(1.5 \mathrm{~W} / \mathrm{cm}^{2}\right.$ $\mathrm{CW}, 3 \mathrm{~min}) . \mathrm{m}$, Control rats. no US. $n=12$. Values are mean \pm SEM.

due to changes in urine flow rather than changes in skin permeation, the rats were catheterized, and the urine volume was recorded. The mean \pm SEM volume of urine secretion during the first $2 \mathrm{~h}$ was $1.50 \pm 0.25 \mathrm{ml}$ for ultrasound-treated rats and $0.84 \pm 0.25 \mathrm{ml}$ for the controls (in the mannitol experiment). The fact that the urination rate of the ultrasound-treated rats was less than twice that of the control rats is thus unable to account for the much higher changes in drug excretion.

The possibility of artifact in the amount of D-mannitol and inulin recovered due to tritium exchange with surrounding aqueous media was also evaluated. The media was assayed as described by Brown et al. (17). No tritium exchange was observed.

Thin-layer chromatography of urine excreted D- $\left[{ }^{3} \mathrm{H}\right]-$ mannitol from ultrasound treated rats was identical to that of untreated rats. The main spot appeared to be $\mathrm{D}-\left[{ }^{3} \mathrm{H}\right]$ mannitol, indicating that the applied mannitol was not decomposed by ultrasound. We did not test the excreted $\left[{ }^{3} \mathrm{H}\right]$ inulin, due to the very small amount secreted, $(<0.03 \%$ of the applied inulin was excreted in $5 \mathrm{~h}$, as compared to $\sim 2 \%$ of mannitol).

To estimate the permeation of physostigmine through the skin, we measured the decrease in blood ChE which depends on the concentration of physostigmine in the blood (Table I)

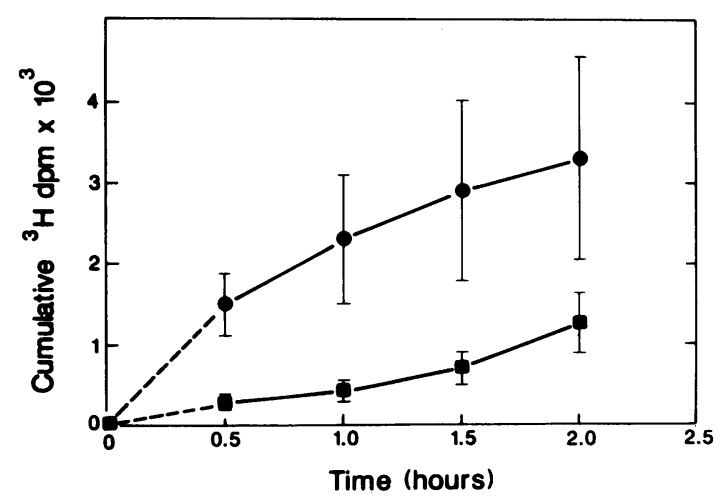

Figure 2. Rats' cumulative radioactive secretion after topical application of $50 \mu \mathrm{l}$ of saturated solution of inulin containing $11 \mu \mathrm{Ci}\left[{ }^{3} \mathrm{H}\right]-$ inulin. $\bullet$, Ultrasound treated rats. $n=4$. $\left(3 \mathrm{~W} / \mathrm{cm}^{2}\right.$ pulsed wave, 5 $\min )$. $n$, Control rats. no US. $n=4$. Values are mean \pm SEM.

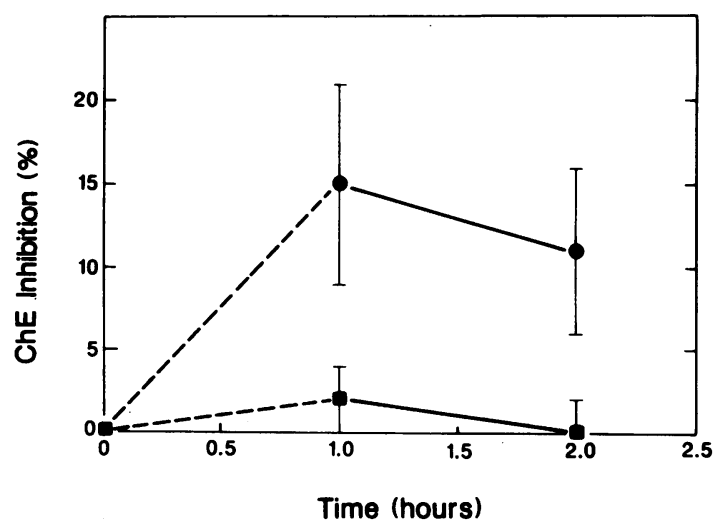

Figure 3. Whole blood $\mathrm{ChE}$ inhibition profile in guinea pigs after topical application of physostigmine ( $30 \% \mathrm{wt} / \mathrm{vol}$ in ethanol). $\bullet$, Ultrasound treated animals. $n=4$. ( $3 \mathrm{~W} / \mathrm{cm}^{2}$ pulsed wave $\left.5 \mathrm{~min}\right) . \mathrm{m}$, Control animals. no US. $n=4$. Values are mean \pm SEM.

(18). The level of ChE inhibition $1 \mathrm{~h}$ following ultrasound application was significantly higher $(P<0.05)$ for both rats and guinea pigs in the ultrasound treated animals than in controls. No significant inhibition of ChE could be detected during the first $2 \mathrm{~h}$ after application of physostigmine to control guinea pigs, while the ultrasound-treated group showed a $15 \pm 5 \%$ decrease in $\mathrm{ChE}$ activity $1 \mathrm{~h}$ after ultrasound application (Fig. 3). $5 \mathrm{~h}$ after ultrasound application, the level of $\mathrm{ChE}$ inhibition was $13 \pm 4 \%$ for both groups (not shown). For the physostigmine-treated rats, the level of $\mathrm{ChE}$ inhibition $1 \mathrm{~h}$ after application was $53 \% \pm 5$ in the ultrasound group and $35 \% \pm 5$ in the control (Fig. 4). To rule out the possibility of ultrasound itself affecting blood ChE activity, ultrasound energy (same conditions as for experimental animals) was applied to a dorsal site of drug-free animals and blood ChE activity was assayed before and after ultrasound treatment. Ultrasound treatment alone had no effect on the level of $\mathrm{ChE}$ inhibition in vivo, nor did it have any effect on the level of $\mathrm{ChE}$ inhibition of physostigmine-treated blood in vitro (Table I), thus indicating that the higher $\mathrm{ChE}$ inhibition is directly related to increased drug penetration. Furthermore, when ultrasound was applied to guinea pigs $1 \mathrm{~h}$ before physostigmine application, there was no difference in $\mathrm{ChE}$ inhibition between

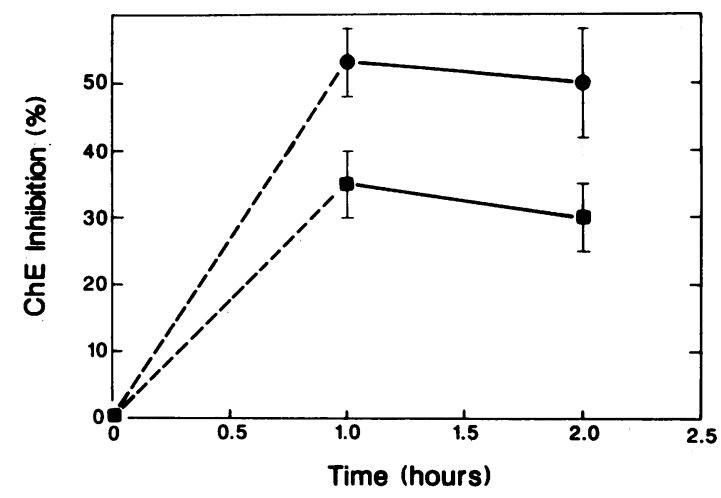

Figure 4. Whole blood ChE inhibition profile in rats after topical application of physostigmine (15\% wt/vol in ethanol). $\bullet$, Ultrasound treated rats. $n=7$. ( $3 \mathrm{~W} / \mathrm{cm}^{2}$ pulsed wave, $5 \mathrm{~min}$ ). $\mathrm{a}$, Control rats. no US. $n=7$. Values are mean \pm SEM. 


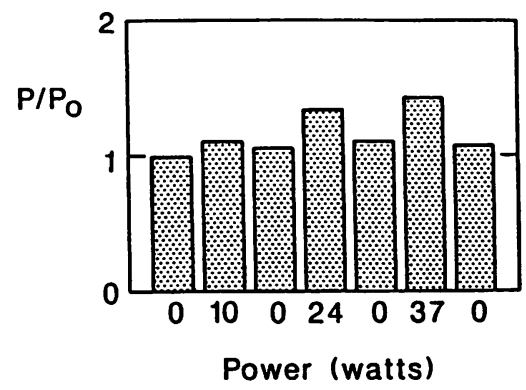

the experimental group and the controls. The increase in surface skin temperature measured immediately after ultrasound application did not exceed $1-2^{\circ} \mathrm{C}$.

Mechanistic studies. To gain insight into the mechanism of ultrasonically enhanced release, synthetic membranes were evaluated in an in vitro diffusion cell. The advantages of using such a defined system involving synthetic membranes rather than skin are that these membranes are very reproducible and their permeability characteristics are well characterized. Therefore individual effects contributing to ultrasound enhanced permeability can be accurately assessed. Studies were conducted to examine both the reversibility of the ultrasound effect and the mechanism of this effect. To evaluate the reversibility of the ultrasound phenomenon, experiments were performed in which the ultrasound was turned on and off alternatively at different power levels and the permeability was calculated. Fig. 5 shows the effect of ultrasound power on the ratio of permeabilities (while exposed to ultrasound to the permeability in unexposed solutions). As can be seen the effect is reversible. The permeabilities of the membranes return to their original values (before ultrasound application), when the output power of the ultrasound was set back to zero.

We considered three factors that might contribute to ultrasound enhanced permeability: mixing, cavitation, and temperature. To examine whether ultrasound might affect a boundary layer surrounding the membranes, and therefore cause higher permeability values, experiments were performed

Table II. The Effect of Mixing Rate on Urea Permeability through Cuprophan Membranes

\begin{tabular}{cc}
\hline Permeability $P * 10^{6}$ & Mixing \\
\hline $\mathrm{cm}^{2} / \mathrm{s}$ & $\mathrm{rpm}$ \\
$1.6 \pm 0.067$ & 0 \\
$1.73 \pm 0.095$ & 300 \\
$1.88 \pm 0.143$ & 600 \\
$1.788 \pm 0.156$ & 900
\end{tabular}

Studies were conducted by placing the membrane in a two-compartment transport cell. A stirrer was placed in the upstream compartment. Urea was analyzed by taking samples from the downstream compartment. For details, see Methods.

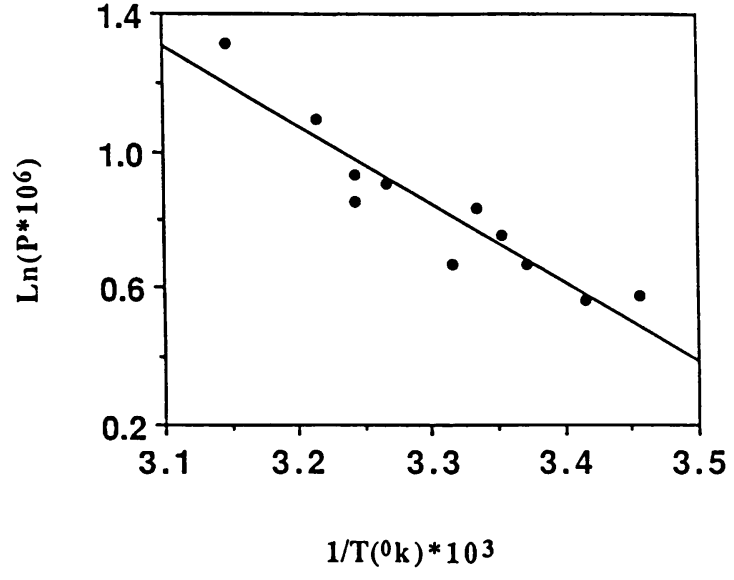

Figure 6. Permeability of urea through cuprophan membranes vs. temperature. Membranes were placed in a two compartment diffusion cell, which was located in a thermostatted environment. The constant temperatures in which the permeability was evaluated were in the range of $17-44^{\circ} \mathrm{C}$.

under controlled mixing rates. A boundary layer is a thin layer of fluid created when a fluid flows over a surface. The velocity in this layer decreases until it is zero at the surface. The boundary layer is an additional resistance for drug transport. The thickness of the layer is inversely proportional to the velocity of flowing fluid and therefore mixing could eliminate a boundary layer and its effect on permeability. Table II summarizes the effect of mixing on the urea permeability through cuprophan membranes. From $600 \mathrm{rpm}$ and greater there is no effect of additional mixing on permeability. An additional factor that could be associated with ultrasound is cavitation (i.e. formation and collapse of gas cavities in the liquid creating shock waves). Pulsation of cavitation bubbles might affect the boundary layer as well as transport by convection. Therefore experiments were conducted in degassed buffer, where cavitation was minimized. The observed enhancement of urea permeability was much smaller: $23 \%$ (at $19.5^{\circ} \mathrm{C}$ and $40 \%$ duty cycle) compared to $55 \%$ in the original buffer at the same conditions and temperature. Heat liberation will eventually be produced within any medium exposed to ultrasonic energy. As mass diffusivity is a function of temperature, urea permeability experiments through cuprophan membranes were performed at defined constant temperatures. The permeability vs. temperature displayed Arrhenius behavior (Fig. 6).

Histology. Comparisons were made between rat skin exposed to ultrasound and controls not exposed to ultrasound. The sections of skin and associated underlying tissues appeared essentially the same and were within normal limits.

\section{Discussion}

One effect, due to ultrasound, observed in this study was the shortening of the lag time, normally associated with all forms of transdermal drug delivery. This was observed in all but the physostigmine-treated rats. The permeability of rat skin to physostigmine solution was relatively high, therefore the lag time phase was not observed perhaps because the sampling 
periods were not frequent enough to detect it. However, although the systemic effect (ChE inhibition) could be observed after $1 \mathrm{~h}$ in both ultrasound-treated and the control rats, the effect was larger in the former group.

The fact that 3-5 min of ultrasound irradiation have shortened the onset time of transdermally applied substances suggests that ultrasound can temporarily and reversibly alter the permeability of skin for both hydrophilic and lipophilic drugs. The reversibility of the ultrasound effect on skin was demonstrated by the observation that ultrasound irradiation of guinea pigs $1 \mathrm{~h}$ before drug application did not change the permeation rate of physostigmine through the skin. This is also supported by the observation that the penetration rate of physostigmine in guinea pigs, $5 \mathrm{~h}$ after ultrasound application, was the same for both ultrasound treated and controls, (as judged by the level of $\mathrm{ChE}$ inhibition) in contrast to the large difference in permeation rate observed right after ultrasound application (Fig. 3).

The small increase in surface skin temperature observed after ultrasound application $\left(1-2^{\circ} \mathrm{C}\right)$, is not likely to cause dramatic changes in skin permeability $(19,20)$. However, ultrasound is known for its ability to cause deep penetrating hyperthermia which may increase diffusivity, solubility of drugs, vasodilation and blood flow, all of which may facilitate drug penetration.

The mechanistic in vitro studies showed that ultrasound can reversibly enhance the permeability of synthetic membranes (Fig. 5). The temperature increase of $1-2^{\circ} \mathrm{C}$ based on the Arrhenius correlation has a relatively very small effect on the permeability. In addition to temperature, other important phenomena involved are mixing and cavitation. Although it's difficult to be certain whether the data obtained in the in vitro experiments with synthetic polymeric membranes are extendable to the in vivo situation with skin, it is likely that since both involve diffusion through membranes, that those factors which ultrasound affects most significantly in vitro (e.g., mixing, cavitation) also play a significant role in vivo (21).

The observed phenomenon that short exposures of ultrasound can alter both the lag time and the permeability of transdermally administered substances suggests a number of future studies. Determining the appropriate ultrasonic frequencies, duty cycles, orientation, and energies to achieve desired effects for different drugs may greatly broaden the range of therapeutic agents that can be administered transdermally. Eliminating the lag phase associated with transdermal dosage forms may eventually broaden the use of these dosage forms to acute as well as chronic indications. Portable ultrasonic triggering devices must be developed, however, if this type of approach is to be generally usable. There has been considerable clinical interest in the use of iontophoresis to affect transdermal drug delivery (5). The current study indicates that ultrasound may also be of value in this regard.

\section{Acknowledgments}

The authors thank R. Chen, O. Lykin, and M. Goldriech for their technical assistance.

This study was supported by National Institutes of Health Grant CM26698 and a grant from the United States-Israel Binational Science Foundation (BSF), Jerusalem, Israel.

\section{References}

1. Maibach, H. I., and R. J. Feldmann. 1967. The effect of DMSO on percutaneous penetration of hydrocortisone and testosterone on man. Ann. NY Acad. Sci. USA 141:423-427.

2. Brown, L. R., and R. Langer. 1988. Transdermal delivery of drugs. Annu. Rev. Med. 39:221-229.

3. Arndts, D., and K. Arndts. 1984. Pharmacokinetics and pharmacodynamics of transdermally administered clonidine. Eur. J. Clin. Pharmacol. 26:79-85.

4. Price, N. M., L. G. Schmitt, G. McGuire, J. E. Shaw, and G. Trobough. 1981. Transdermal scopolamine in the prevention of motion sickness at sea. Clin. Pharmacol. Ther. 29:414-419.

5. Kari, B. 1986. Control of blood glucose levels in alloxan-diabetic rabbits by iontophoresis of insulin. Diabetes. 35:217-221.

6. Cleary, G. W. 1984. Transdermal controlled release systems. In Medical Applications of Controlled Release. Vol. 1. R. S. Langer and D. L. Wise, editors. CRC Press, Inc, Boca Raton, FL. 203-253.

7. Kost, J., K. Leong, and R. Langer. 1986. Ultrasonic modulated drug delivery systems. In Polymers in Medicine, Biology and Pharmaceutical Applications II. E. Chiellini, editor. Plenum Press, New York/London. 376-396.

8. McElnay, J. C., M. P. Mathews, R. Harland, and D. F. McCafferty. 1985. The effect of ultrasound on the percutanous absorption of lignocaine, Br. J. Clin. Pharmacol. 20:421-424.

9. Fellinger, K., and J. Schmid. 1954. Klinik und Therapie des chronischen, Gelenkreumatismus. Maudrich, Vienna, Austria. 549-554.

10. Skauen, D. M., and G. M. Zentner. 1984. Phonophoresis, Int. J. Pharmacol. 20:235-245.

11. Gutman, Y., C. W. Gattschalk, and W. T. Lassiter. 1965. Macropuncture study of inulin absorption in the rat kidney. Science (Wash. DC). 147:753-754.

12. Weiner, I. M., and G. H. Mudge. 1985. Diuretics and other agents employed in the mobilization of edema fluid. In The Pharmacological Basis of Therapeutics. 7th ed. L. Goodman, A. Gilman, T. W. Rall, and F. Murad, editors. McMillan Publishing Co., New York. 887-889.

13. Taylor, $P$. 1985. Anticholinesterase agents. In The Pharmacological Basis of Therapeutics. 7th ed. L. Goodman, A. Gilman, T. W. Rall, and F. Murad, editors. McMillan Publishing Co., New York. 110-129.

14. Thal, L. J., P. A. Fuld, D. M. Masur, N. S. Sharpless, and P. Davies. 1983. Oral physostigmine and lecithin improve memory in Alzheimer's disease, Psychopharmacol. Bull. 19:454-456.

15. March, W. H., B. Fingerhut, and H. Miller. 1965. Automated and manual direct methods for the determination of blood urea. Clin. Chem. 11:624-627.

16. Johnson, C. D., and R. L. Russell. 1975. A rapid simple radiometric assay for cholinesterase, suitable for multiple determinations. Anal. Biochem. 64:229-238.

17. Brown, L. R., C. L. Wei, and R. Langer. 1983. In vivo and in vitro release of macromolecules from polymeric drug delivery systems. J. Pharmacol. Sci. 72:1181-1185.

18. Aldridge, W. N., and E. Reiner. 1972. Enzyme Inhibitors as Substrates. North Holland Publishing Co., Amsterdam/London.

19. Knutson, K., R. O. Potts, D. B. Guzek, G. M. Golden, J. E. McKie, W. J. Lambert, and W. I. Higuchi. 1985. Macro and molecular physical-chemical considerations in understanding drug transport in the stratum corneum. J. Controlled Release. 2:67-87.

20. Flynn, G. L. 1985. Mechanism of percutanous absorption from physicochemical evidence. In Percutanous Absorption. R. L. Bronaugh, and H. I. Maibach, editors. Marcel Dekker, Inc., New York/ Basel. 33.

21. Haar, G. R., and S. Daniels. 1981. Evidence for ultrasonically induced cavitation in vivo. Phys. Med. Biol. 26:1145-1149. 